\title{
Dynamics towards the Feigenbaum attractor
}

\author{
A. Robledo \\ Instituto de Física, Universidad Nacional Autónoma de México, \\ Apartado postal 20-364, México 01000 D.F., México* \\ L. G. Moyano \\ Telefónica I+D, Emilio Vargas 6, 28043, Madrid, Spain ${ }^{\dagger}$
}

(Received on 14 January, 2009)

\begin{abstract}
As an essential component in the demonstration of an atypical, $q$-deformed, statistical mechanical structure in the dynamics of the Feigenbaum attractor we expose, at a previously unknown level of detail, the features of the dynamics of trajectories that either evolve towards the Feigenbaum attractor or are captured by its matching repellor. Amongst these features are the following: i) The set of preimages of the attractor and of the repellor are embedded (dense) into each other. ii) The preimage layout is obtained as the limiting form of the rank structure of the fractal boundaries between attractor and repellor positions for the family of supercycle attractors. iii) The joint set of preimages for each case form an infinite number of families of well-defined phase-space gaps in the attractor or in the repellor. iv) The gaps in each of these families can be ordered with decreasing width in accord to power laws and are seen to appear sequentially in the dynamics generated by uniform distributions of initial conditions. v) The power law with log-periodic modulation associated to the rate of approach of trajectories towards the attractor (and to the repellor) is explained in terms of the progression of gap formation. vi) The relationship between the law of rate of convergence to the attractor and the inexhaustible hierarchy feature of the preimage structure is elucidated. We discuss the function of these properties in the atypical thermodynamic framework existing at the period-doubling transition to chaos.
\end{abstract}

Keywords: Feigenbaum attractor, Supercycles, Convergence to attractor, Log-periodic oscillation

\section{INTRODUCTION}

A fundamental question in statistical physics is to know whether the structure of ordinary equilibrium statistical mechanics falters when its fundamental properties, phase space mixing and ergodicity, breakdown. The chaotic dynamics displayed by dissipative nonlinear systems, even those of low dimensionality, possesses these two crucial conditions, and acts in accordance with a formal structure analogous to that of canonical statistical mechanics, in which thermodynamic concepts meet their dynamical counterparts [1]. At the transition between chaotic and regular behaviors, classically represented by the Feigenbaum attractor [1], the Lyapunov exponent vanishes and chaotic dynamics turns critical. Trajectories cease to be ergodic and mixing; they retain memory back to their initial positions and fluctuate according to complex deterministic patterns [2]. Under these conditions it is of interest to check up whether the statistical-mechanical structure subsists, and if so, to examine if it is unchanged or if it has acquired a new form.

With this purpose in mind, the exploration of possible limits of validity of the canonical statistical mechanics, an ideal model system is a one-dimensional map at the transition between chaotic and regular behaviors, represented by wellknown critical attractors, such as the Feigenbaum attractor. So far, recent studies [3] have concentrated on the dynamics inside the attractor and have revealed that these trajectories obey remarkably rich scaling properties not known previously at this level of detail [4]. The results are exact and clarify [4] the relationship between the original modification [5] [2] of the thermodynamic approach to chaotic attractors [6] [7] [8]

\footnotetext{
${ }^{*}$ Electronic address: robledo@fisica. unam.mx

${ }^{\dagger}$ Electronic address: moyano@tid.es
}

for this type of incipiently chaotic attractor, and some aspects of the $q$-deformed statistical mechanical formalism [9] [10] [11]. The complementary part of the dynamics, that of advance on the way to the attractor, has, to our knowledge, not been analyzed, nor understood, with similar degree of thoroughness. The process of convergence of trajectories into the Feigenbaum attractor poses several interesting questions that we attempt to answer here and elsewhere [12] based on the comprehensive new knowledge presented below. Prominent amongst these questions is the nature of the connection between the two sets of dynamical properties, within and outside the attractor. As it turns out [12], these two sets of properties are related to each other in a statistical-mechanical manner, i.e. the dynamics at the attractor provides the configurations in a partition function while the approach to the attractor is described by an entropy obtained from it. As we show in [12], this statistical-mechanical property conforms to a $q$-deformation [9] of the ordinary exponential weight statistics.

Trajectories inside the attractor visit positions forming oscillating log-periodic patterns of ever increasing amplitude. However, when the trajectories are observed only at specified times, positions align according to power laws, or $q$ exponential functions that share the same $q$-index value [4] [10]. Further, all such sequences of positions can be shifted and seen to collapse into a single one by a rescaling operation similar to that observed for correlations in glassy dynamics, a property known as 'aging' [10] [13]. The structure found in the dynamics is also seen to consist of a family of Mori's $q$-phase transitions [2], via which the connection is made between the modified thermodynamic approach and the $q$-statistical property of the sensitivity to initial conditions [4] [10]. On the other hand, a foretaste of the nature of the dynamics outside the critical attractor can be appreciated by considering the dynamics towards the so-called supercycles, the family of periodic attractors with Lyapunov expo- 
nents that diverge towards minus infinity. This infinite family of attractors has as accumulation point the transition to chaos, which for the period-doubling route is the Feigenbaum attractor. As described in Ref. [14], the basins of attraction for the different positions of the cycles develop fractal boundaries of increasing complexity as the period-doubling structure advances towards the transition to chaos. The fractal boundaries, formed by the preimages of the repellor, display hierarchical structures organized according to exponential clusterings that manifest in the dynamics as sensitivity to the final state and transient chaos. The hierarchical arrangement expands as the period of the supercycle increases [14].

Here we present details on the general procedure followed by trajectories to reach the Feigenbaum attractor, and its complementary repellor. We consider an ensemble of uniformly distributed initial conditions $x_{0}$ spanning the entire phase space interval. This is a highly structured process encoded in sequences of positions shared by as many trajectories with different $x_{0}$. There is always a natural dynamical ordering in the $x_{0}$ as any trajectory of length $t$ contains consecutive positions of other trajectories of lengths $t-1, t-2$, etc. with initial conditions $x_{0}^{\prime}, x_{0}^{\prime \prime}$, etc. that are images under repeated map iterations of $x_{0}$. The initial conditions form two sets, dense in each other, of preimages each the attractor and the repellor. There is an infinite-level structure within these sets that, as we shall see, is reflected by the infinite number of families of phase-space gaps that complement the multifractal layout of both attractor and repellor. These families of gaps appear sequentially in the dynamics, beginning with the largest and followed by other sets consisting of continually increasing elements with decreasing widths. The number of gaps in each set of comparable widths increases as $2^{k}, k=0,1, \ldots$ and their widths can be ordered according to power laws of the form $\alpha^{-k}$, where $\alpha$ is Feigenbaum's universal constant $\alpha \simeq 2.5091$. We call $k$ the order of the gap set. Furthermore, by considering a fine partition of phase space, we determine the overall rate of approach of trajectories towards the attractor (and to the repellor). This rate is measured by the fraction of bins $W(t)$ still occupied by trajectories at time $t$ [15]. The power law with log-periodic modulation displayed by $W(t)[15]$ is explained in terms of the progression of gap formation, and its self-similar features are seen to originate in the unlimited hierarchy feature of the preimage structure.

Before proceeding to expand our description in the following sections we recall [16] the general definition of the interval lengths or diameters $d_{N, m}$ that measure the bifurcation forks that form the period-doubling cascade sequence in the logistic map $f_{\mu}(x)=1-\mu x^{2},-1 \leq x \leq 1,0 \leq \mu \leq 2$. These quantities are measured when considering the superstable periodic orbits of lengths $2^{N}$, i.e. the $2^{N}$-cycles that contain the point $x=0$ at $\bar{\mu}_{N}<\mu_{\infty}$, where $\mu_{\infty}=1.401155189 \ldots$ is the value of the control parameter $\mu$ at the period-doubling accumulation point [1]. The positions of the limit $2^{\infty}$-cycle constitute the Feigenbaum attractor. The $d_{N, m}$ in these orbits are defined (here) as the (positive) distances of the elements $x_{n}$, $m=0,1,2, \ldots, 2^{N}-1$, to their nearest neighbors $f_{\bar{\mu}_{N}}^{\left(2^{N-1}\right)}\left(x_{m}\right)$, i.e.

$$
d_{N, m} \equiv\left|f_{\bar{\mu}_{N}}^{\left(m+2^{N-1}\right)}(0)-f_{\bar{\mu}_{N}}^{(m)}(0)\right| .
$$

For large $N, d_{N, 0} / d_{N+1,0} \simeq \alpha$. Notice that infinitely many other sequences of superstable attractors appear at the perioddoubling cascades within the windows of periodic attractors for values of $\mu>\mu_{\infty}$. At each of these period-doubling accumulation points occur replicas of the Feigenbaum attractor. We present explicit results for the logistic map, that has a quadratic maximum, but the results are easily extended to unimodal maps with general nonlinearity $z>1$.

Central to our discussion is the following broad property: Time evolution at $\mu_{\infty}$ from $t=0$ up to $t \rightarrow \infty$ traces the perioddoubling cascade progression from $\mu=0$ up to $\mu_{\infty}$. Not only is there a close resemblance between the two developments but also quantitative agreement. For instance, the trajectory inside the Feigenbaum attractor with initial condition $x_{0}=0$, the $2^{\infty}$-supercycle orbit, takes positions $x_{t}$ such that the distances between appropriate pairs of them reproduce the diameters $d_{N, m}$ defined from the supercycle orbits with $\bar{\mu}_{N}<\mu_{\infty}$. See Fig. 1, where the absolute value of positions and logarithmic scales are used to illustrate the equivalence. This property has been key to obtain rigorous results for the sensitivity to initial conditions for the Feigenbaum attractor [3], [10].
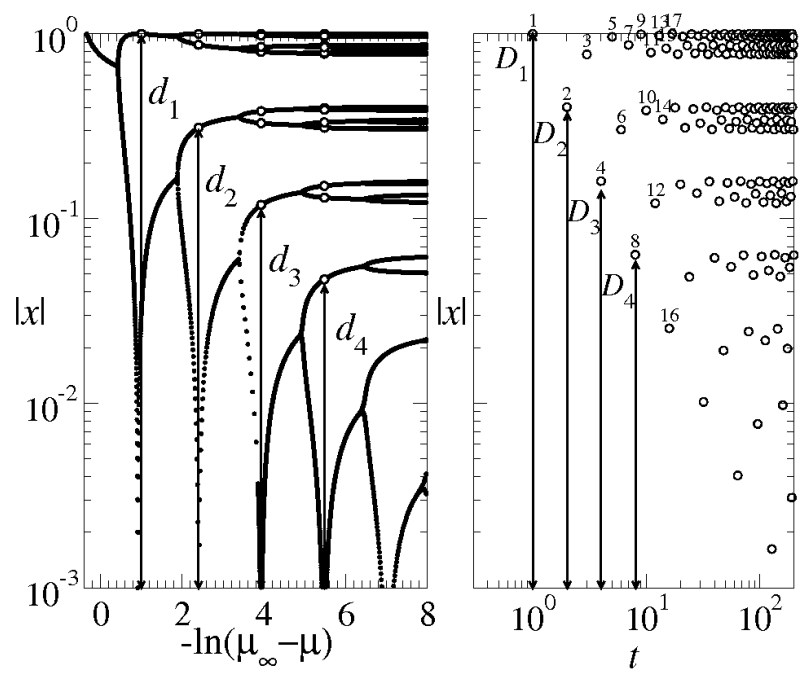

FIG. 1: Left panel: Absolute value of attractor positions for the logistic map $f_{\mu}(x)$ in logarithmic scale as a function of the logarithm of the control parameter difference $\mu_{\infty}-\mu$. Right panel: Absolute value of trajectory positions for the logistic map $f_{\mu}(x)$ at $\mu_{\infty}$ with initial condition $x_{0}=0$ in logarithmic scale as a function of the logarithm of time $t$, also show by the numbers close to the circles. The arrows indicate the equivalence between the diameters $d_{N}$ in the left panel, and position differences $D_{N}$ with respect to $x_{0}=0$ in the right panel.

\section{PREIMAGE STRUCTURE OF ATTRACTOR AND REPELLOR}

A convenient way to visualize how the preimages for the Feigenbaum attractor and repellor are distributed and organized is to consider the simpler arrangements for the preimages of the supercycles' attractors and repellors. These arrangements have been characterized in detail recently [14] for supercycles of small periods $2^{N}, N=1,2, \ldots$, and it was observed how they become more complicated very rapidly as 
$N$ grows [14]. For $N=1$ the preimages of the attractor belong to only two basins, one for each position of the attractor, separated by the positions of the fixed-point repellor and its single preimage. For $N=2$ the attractor preimages are distributed into four basins, one pair of them separated from the other pair by a fractal boundary built around the positions of the first or 'old' $N=1$ repellor and its preimage. This fractal boundary consists of the infinite number of preimages that the old repellor has acquired and this in turn cluster exponentially around it and around its 'old' preimage. For $N=3$ the attractor preimages are distributed into eight basins, four of the basins are separated from the remaining four by a more complex fractal boundary sector, built again about the positions of the old $N=1$ repellor and its old preimage. This sector is made of an infinite number of replicas of the fractal boundary for the case with $N=2$, the increasingly more numerous preimages of the old repellor form now an infinite set of clusters, grouping themselves exponentially around it and around its old preimage. The basins for the positions of the attractor are also separated from each other in pairs via other fractal boundary segments with the characteristics of the $N=2$ case, structures that appear centered now around the next generation of repellors (two) and their first preimages (two). In the panels of Fig. 2 we show the relative times $t_{f}$ required to reach the attractor as a function of the initial condition $x_{0}$ for the supercycles with $N=1,2$, and 3 , respectively. The logarithmic horizontal scale in these figures relative to the position of the old repellor reveals the main characteristic of the preimage structure for each case. These figures illustrate our description above. See Ref. [14] for the definition of $t_{f}$ and further details.

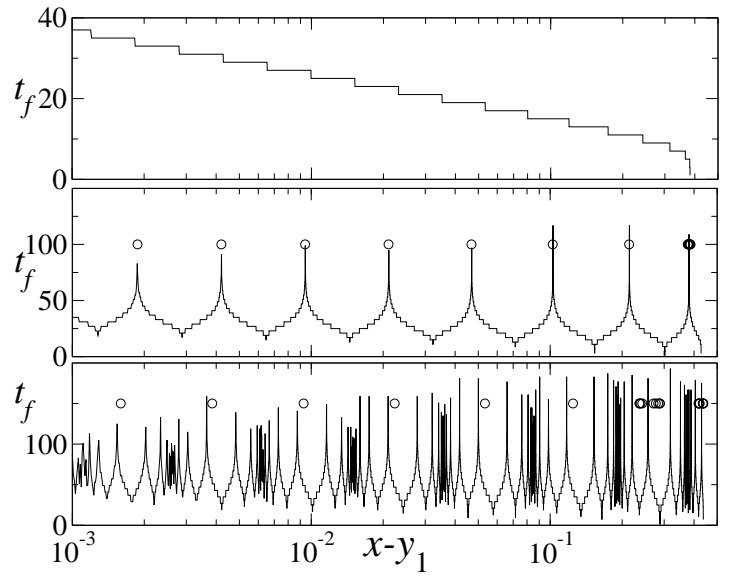

FIG. 2: Time of flight $t_{f}(x)$, the number of iterations necessary for a trajectory with initial condition at $x$ to reach an attractor position as a function of the distance, in logarithmic scale, between $x$ and the repellor position at $y_{1}$. The top panel is for the twice-iterated map $f_{\bar{\mu}_{1}}^{(2)}(x), \bar{\mu}_{1}=1, y_{1} \simeq 0.6180340$. The middle panel for $f_{\bar{\mu}_{2}}^{(4)}(x)$, $\bar{\mu}_{2} \simeq 1.31070264, y_{1} \simeq 0.571663$. And the bottom panel for $f_{\bar{\mu}_{3}}^{(8)}(x)$, $\bar{\mu}_{3} \simeq 1.38154748, y_{1} \simeq 0.56264475$. The values of $x$ near the peaks (open circles) correspond to initial conditions very close to the repellor preimages. See text.

As the period $2^{N}$ increases the preimage structures for the attractor and repellor become more and more involved, with the appearance of new features made up of an infinite repeti- tion of building blocks. Each of these new blocks is equivalent to the more dense structures present in the previous $2^{N-1}$ case. In addition all other structures in the earlier $2^{N-2}, \ldots$, $2^{1}$ cases are still present. Thus a hierarchical organization of preimages is built upon as $N$ increases, so that the preimage layout for the Feigenbaum attractor and repellor is obtained as the limiting form of the rank structure of the fractal boundaries between the finite period attractor position basins. The fractal boundaries consist of sub-basins of preimages for the attractor positions separated by preimages of the repellor positions. As $N$ increases, the sizes of these sub-basins decrease while their numbers increase and the fractal boundaries cover a progressively larger part of total phase space. See Fig. 2 and also Figs. 3 and 6 of Ref. [14].

Interestingly, the sizes of all boundary sub-basins vanish in the limit $N \rightarrow \infty$, and the preimages of both attractor and repellor positions become two sets - with dimension equal to the dimension of phase space - dense in each other. In the limit $N \rightarrow \infty$ there is an attractor preimage between any two repellor preimages and the other way round. (The attractor and repellor are two multifractal sets with dimension $d_{f} \simeq 0.538 \ldots$ [16]). To visualize this limiting situation consider that the positions for the repellor and their first preimages of the $2^{N}$-th supercycle appear located at the inflection points of $f_{\bar{\mu}_{N}}^{\left(2^{N}\right)}(x)$, and it is in the close vicinity of them that the mentioned fractal boundaries form. To illustrate how the sets of preimage structures for the Feigenbaum attractor and repellor develop we plot in Fig. 3 the absolute value of $\ln \left|d f_{\bar{\mu}_{N}}^{\left(2^{N}\right)} / d x\right|$ for $N=1,2, \ldots, 4$ vs. $x$. The maxima in this curve correspond to the inflection points of $f_{\bar{\mu}_{N}}^{\left(2^{N}\right)}(x)$ at which the repellor positions or their first preimages are located. As shown in Fig. 3, when $N$ increases the number of maxima proliferate at a rate faster than $2^{N}$.

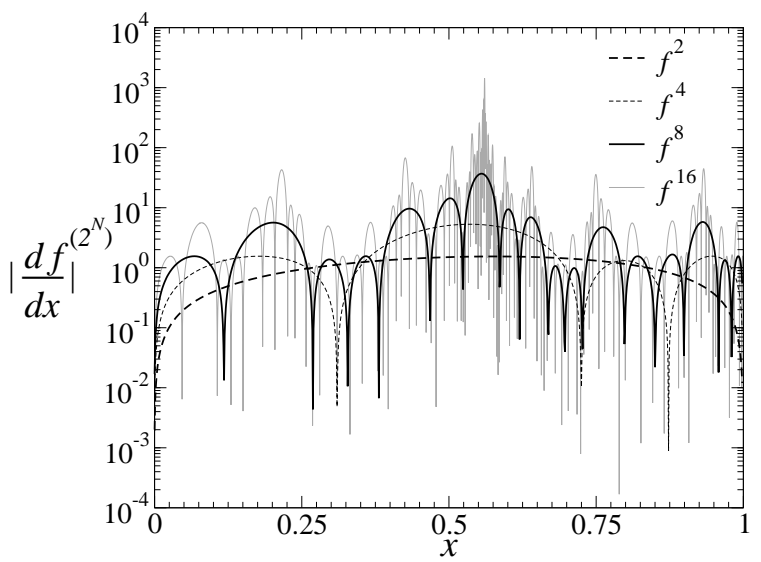

FIG. 3: The absolute value of $d f_{\bar{\mu}_{N}}^{\left(2^{N}\right)}(x) / d x$, for $N=1,2,3$ and 4 , in logarithmic scale as a function of $x$ in the interval $0 \leq x \leq 1$. The proliferation of maxima conveys the development of the hierarchical structure of repellor preimages. See text. 


\section{SEQUENTIAL OPENING OF PHASE SPACE GAPS}

One way wherein the preimage structure described above manifests in the dynamics is via the successive formation of phase space gaps that ultimately give rise to the attractor and repellor multifractal sets. In order to observe explicitly this process we consider an ensamble of initial conditions $x_{0}$ spread out uniformly across the interval $-1 \leq x_{0} \leq 1$ and keep track of their positions at subsequent times. In Figs. 4 to 6 we illustrate the outcome for the supercycles of periods $2^{2}, 2^{3}$ and $2^{4}$, respectively, where we have plotted the time evolution of an ensemble composed of 10000 trajectories. In the left panel of each figure we show the absolute value of the positions $\left|x_{t}\right|$ vs time $t$, while, for comparison purposes, in the right panel we show the absolute value of $|x|$ both vs $f_{\bar{\mu}_{N}}^{\left(2^{N}\right)}(x)$ and $v s\left|d f_{\bar{\mu}_{N}}^{\left(2^{N}\right)} / d x\right|$ to facilitate identification of the attractor and repellor positions. The labels $k=1,2,3, \ldots$ indicate the order of the gap set (or equivalently the order of the repellor generation set [14]). In Fig. 4 (with $\mu=\bar{\mu}_{2}$ ) one observes a large gap opening first that contains the old repellor $(k=0)$ in its middle region and two smaller gaps opening afterward that contain the two repellors of second generation $(k=1)$ once more around the middle of them. In Fig. 5 (with $\mu=\bar{\mu}_{3}$ ) we initially observe the opening of a primary and the two secondary gaps as in the previous $\mu=\bar{\mu}_{2}$ case, but subsequently four new smaller gaps open each around the third generation of repellor positions $(k=2)$. In Fig. 6 (with $\mu=\bar{\mu}_{4}$ ) we observe the same development as before, however at longer times eight additional and yet smaller gaps emerge each around the fourth generation of repellor positions $(k=3)$. Naturally, this process continues indefinitely as $N \rightarrow \infty$ and illustrates the property mentioned before for $\mu_{\infty}$, that time evolution at fixed control parameter value resembles progression from $\mu=0$ up to, in this paragraph, $\bar{\mu}_{N}$. It is evident in all Figs. 4 to 6 that the closer the initial conditions $x_{0}$ are to the repellor positions, the longer times it takes for the resultant trajectories to clear the gap regions. This intuitively evident feature is essentially linked to the knowledge we have gained about the fractal boundaries of the preimage structure, and the observable 'bent over' portions of these distinct trajectories in the figures correspond to their passage across the boundaries. (Since the ensemble used in the numerical experiments is finite there appear only a few such trajectories in Figs. 4 to 6).

To facilitate a visual comparison between the process of gap formation at $\mu_{\infty}$ and the dynamics inside the Feigenbaum attractor (as illustrated by the trajectory in Fig. 1b) we plot in Fig. 7 the time evolution of the same ensemble composed of 10000 trajectories with $\mu=\mu_{\infty}$. We use this time logarithmic scales for both $\left|x_{t}\right|$ and $t$ and then superpose on the evolution of the ensemble the positions for the trajectory starting at $x_{0}=0$. It is clear from this figure that the larger gaps that form consecutively have all the same width in the logarithmic scale of the plot and therefore their actual widths decrease as a power law, the same power law followed, for instance, by the position subsequence $x_{t}=\alpha^{-N}, t=2^{N}, N=0,1,2, \ldots$ for the trajectory inside the attractor starting at $x_{0}=0$. This set of gaps develops in time beginning with the largest one containing the $k=0$ repellor, then followed by a second gap, one of a set of two gaps associated to the $k=1$ repellor, next

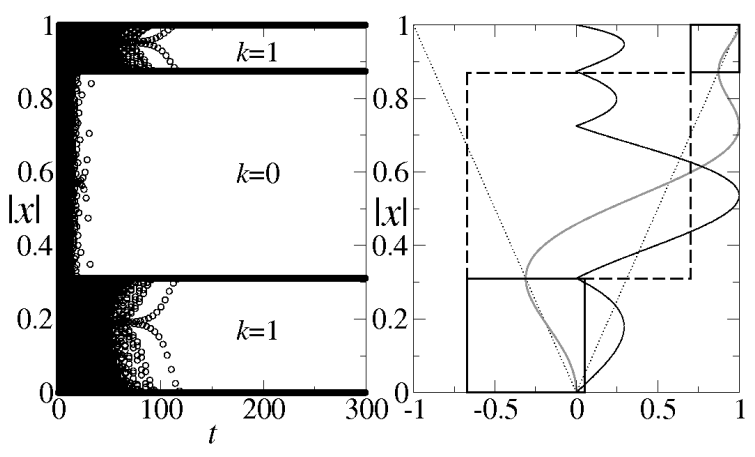

FIG. 4: Phase-space gap formation for $\mu=\bar{\mu}_{2}$. Left panel: time evolution of a uniform ensemble of 10000 trajectories as a function of $|x|$ (black areas and open circles). The values of the index $k$ label the order of the gap set. Right panel: Turned around plots of $f_{\bar{\mu}_{2}}^{(4)}(x)$ (grey) and $\left|d f_{\bar{\mu}_{2}}^{(4)}(x) / d x\right|($ black $)$ vs $|x|$ as guides for the identification of attractor and repellor positions.

a third gap, one gap of a set of four gaps associated to the $k=2$ repellor, and so forth. The locations of this specific family of consecutive gaps advance monotonically towards the most sparse region of the multifractal attractor located at $x=0$. The remaining gaps formed at each stage converge, of course, to locations near other regions of the multifractal but are not easily seen in Fig. 7 because of the specific way in which this has been plotted (and because of the scale used). In Fig. 8 we plot the same data differently, with the variable $\ln |x|$ replaced by $\ln |1-x|$ where now another specific family of gaps, one for each value of $k=0,1,2, \ldots$, appears all with the same width in the logarithmic scale, their actual widths decrease now as $\alpha^{-2 N}, N=0,1,2, \ldots$ The locations of this second family of consecutive gaps advance monotonically towards the most crowded region of the multifractal attractor located at $x=1$. The time necessary for the formation of successive gaps of order $k=0,1,2, \ldots$, increases as $2^{k}$ because the duration of equivalent movements of the trajectories across the corresponding preimage structures involve the $2^{k}$-th composed function $f_{\bar{\mu}_{N}}^{\left(2^{k}\right)}(x)$.

\section{SCALING FOR THE RATE OF CONVERGENCE TO THE ATTRACTOR AND REPELLOR}

There is [15] an all-inclusive and uncomplicated way to measure the rate of convergence of an ensemble of trajectories to the attractor (and to the repellor) that consists of a single time-dependent quantity. A partition of phase space is made of $N_{b}$ equally sized boxes or bins and a uniform distribution, of $N_{c}$ initial conditions placed along the interval $-1 \leq x \leq 1$, is considered again. The number $r$ of trajectories per box is $r=N_{c} / N_{b}$. The quantity of interest is the number of boxes $W(t)$ that contain trajectories at time $t$. This is shown in Fig. 9 in logarithmic scales for the first five supercycles of periods $2^{1}$ to $2^{5}$ where we can observe the following features: In all cases $W(t)$ shows a similar initial nearly constant plateau and a final well-defined decay to zero. As it can be observed in the left panel of Fig. 9 the duration of the final decay grows (ap- 


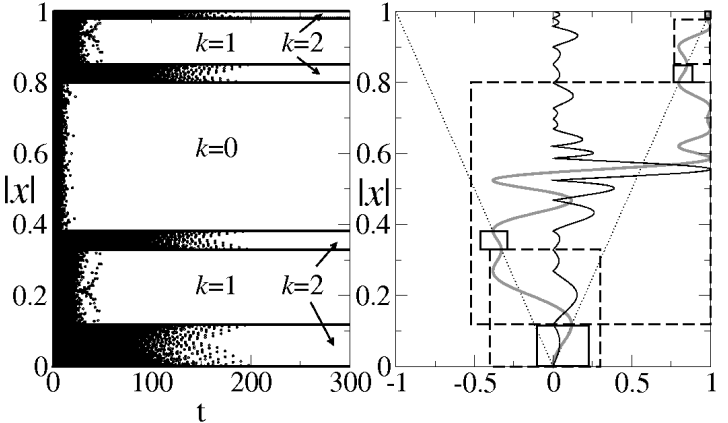

FIG. 5: Phase-space gap formation for $\mu=\bar{\mu}_{3}$. Left panel: time evolution of a uniform ensemble of 10000 trajectories as a function of $|x|$ (black areas and open circles). The values of the index $k$ label the order of the gap set. Right panel: Turned around plots of $f_{\bar{\mu}_{3}}^{(8)}(x)$ (grey) and $\left|d f_{\bar{\mu}_{3}}^{(8)}(x) / d x\right|$ (black) vs $|x|$ as guides for the identification of attractor and repellor positions.

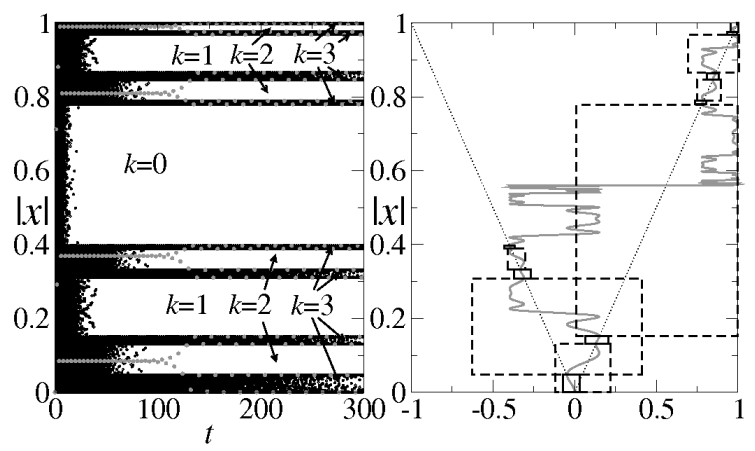

FIG. 6: Phase-space gap formation for $\mu=\bar{\mu}_{4}$. Left panel: time evolution of a uniform ensemble of 10000 trajectories as a function of $|x|$ (black areas and open circles). The values of the index $k$ label the order of the gap set. Right panel: Turned around plots of $f_{\bar{\mu}_{4}}^{(16)}(x)$ (grey) and $\left|d f_{\bar{\mu}_{4}}^{(16)}(x) / d x\right|$ (black) vs $|x|$ as guides for the identification of attractor and repellor positions.

proximately) proportional to the period $2^{N}$ of the supercycle. There is an intermediate slow decay of $W(t)$ that develops as $N$ increases with duration also (just about) proportional to $2^{N}$. For the shortest period $2^{1}$ there is no intermediate feature in $W(t)$, this appears first for period $2^{2}$ as a single dip and expands with one undulation every time $N$ increases by one unit. The expanding intermediate regime exhibits the development of a power-law decay with the logarithmic oscillations characteristic of discrete scale invariance [17]. Clearly, the manifestation of discrete invariance is expected to be associated to the period-doubling cascade. In the right panel of Fig. 9 we show a superposition of the five curves in Fig. 9 (left panel) obtained via rescaling of both $W(t)$ and $t$ for each curve according to repeated scale factors.

The limiting form $W(t)$ for $N \rightarrow \infty$ is shown in the left panel of Fig. 10 for various values of $r$ while in its right panel we show, for $r=100$, a scale amplification of $W(t)$ with the same factors employed in Fig. 9 for the supercycles with small periods. The behavior of $W(t)$ at $\mu_{\infty}$ was originally presented in Ref. [15] where the power law exponent $s$ and

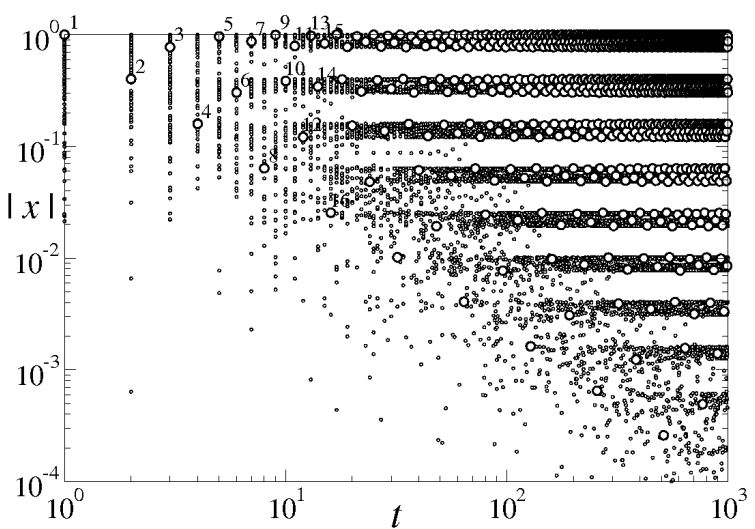

FIG. 7: Phase-space gap formation for $\mu=\mu_{\infty}$. The black dots correspond to time evolution of a uniform ensemble of 10000 trajectories as a function of $|x|$ vs $t$, both in logarithmic scales. The open circles are the positions, labeled by the times at which they are reached, for the trajectory inside the Feigenbaum attractor with initial condition $x_{0}=0$, same as right panel in Fig. 1 .

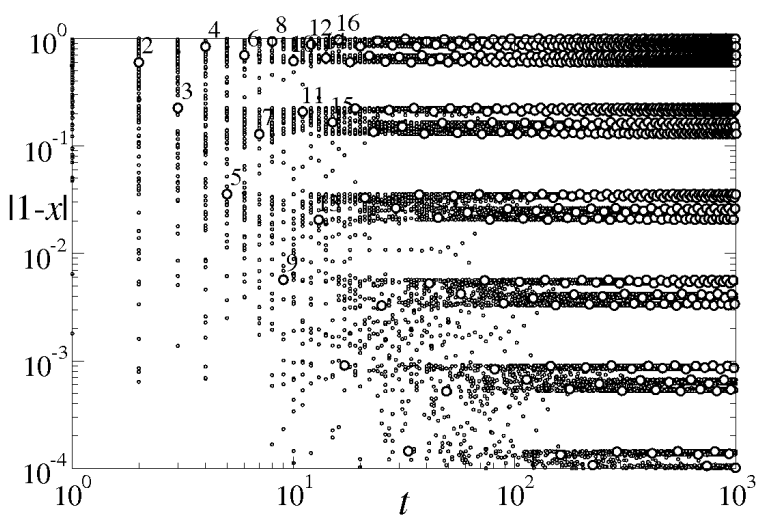

FIG. 8: Same as Fig. 7 but with replacement of $|x|$ by $|1-x|$. Notice the change in slope with respect to Fig. 7 in the opening of gaps and in the layout of the positions for the trajectory inside the attractor.

the logarithmic oscillation parameter $\Lambda$ in

$$
W(t) \simeq h\left(\frac{\ln t}{\ln \Lambda}\right) t^{-s}
$$

were obtained numerically with a precision that corresponds to $r=10$. In Eq. (2) $h(x)$ is a periodic function and $\Lambda$ is the scaling factor between the periods of two consecutive oscillations. More recently, in Ref. [18] it was pointed out that numerical estimates of $W(t)$ are subject to large finite-size corrections, and, also, that $W(t)$ should scale with the intervals in the triadic cantor set construction of the Feigenbaum attractor [18][19], from which the value for $s \cong 0.800138194$ is reported. The values for the rescaling factors in our Figs. 9 and 10 suffer from these large finite size effects due to the relatively small values of $r$ used in the calculations. This is evident since the time scaling factor obtained from these data differs $10 \%$ from the exact value of $\Lambda=2$ implied by the discrete scale invariance property created by the period-doubling cascade. In Fig. 11 we show the rate $W(t)$ and the superposition of repeated amplifications of itself (as in the right panel 
of Fig. 10) for increasing values of $N_{c}$. We find that the scaling factor $\Lambda$ converges to its limit $\Lambda=2$.

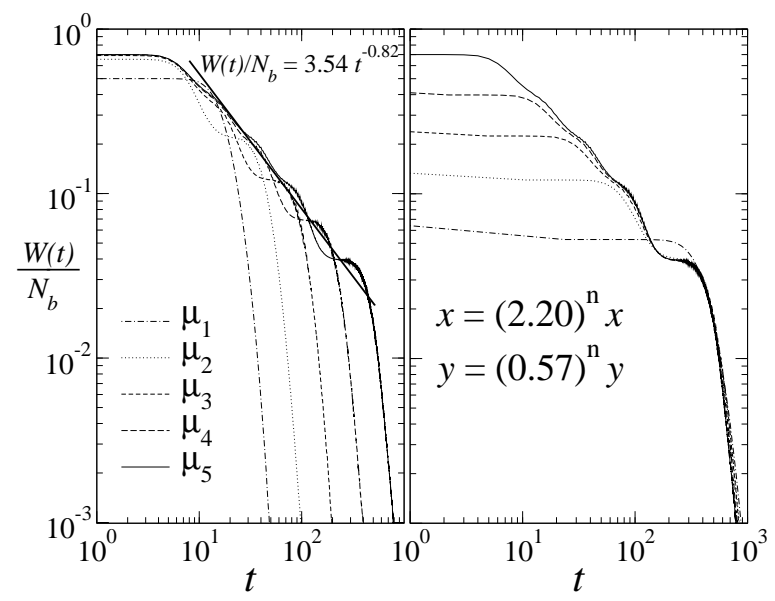

FIG. 9: Left panel: The rate $W(t)$, divided by the number of boxes $N_{b}$ employed, of approach to the attractor for the supercycles of periods $2^{N}, N=1,2,3,4$ and 5 in logarithmic scales. The expression shown corresponds to the power-law decay of the developing logarithmic oscillations. Right panel: Superposition of the five curves for $W(t)$ in the left panel via $n$-times repeated rescaling factors shown for the horizontal $x$ and vertical $y$ axis.

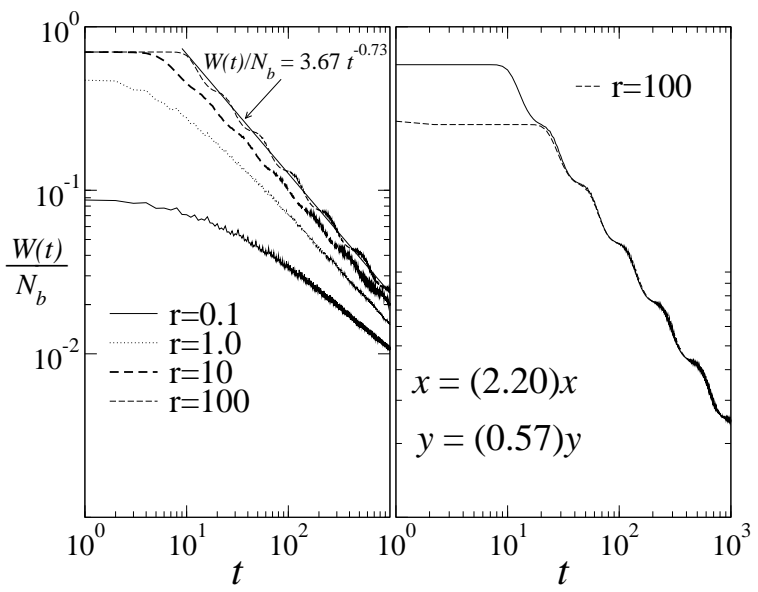

FIG. 10: Left panel: The rate $W(t)$ of approach to the Feigenbaum attractor at $\mu=\mu_{\infty}$ in logarithmic scales. The curves correspond to the values given for the number $r$ of trayectories per box, and the expression shown corresponds to the power-law decay with logarithmic oscillations. Right panel: Superposition of $W(t)$, for $r=100$, with itself via the rescaling shown (the same as in Fig. 8) for the horizontal $x$ and vertical $y$ axis.

We are now in a position to appreciate the dynamical mechanism at work behind the features of the decay rate $W(t)$. From our previous discussion we know that every time the period of a supercycle increases from $2^{N-1}$ to $2^{N}$ by shifting the control parameter value from $\bar{\mu}_{N-1}$ to $\bar{\mu}_{N}$, the preimage structure advances one stage of complication in their hierarchy. Along with this, and in relation to the time evolution of the ensemble of trajectories, an additional set of $2^{N}$ smaller phase-space gaps develops and also a further oscillation takes place in the corresponding rate $W(t)$ for finite-period attractors. At $\mu=\mu_{\infty}$ time evolution tracks the period-doubling cascade progression and the flow of trajectories undergo, every time $t$ increases from $2^{N-1}$ to $2^{N}$, equivalent passages across stages in their itinerary through the preimage ladder structure, in the development of phase-space gaps, and in logarithmic oscillations in $W(t)$. In Fig. 12 we show the correspondence between the latter features quantitatively. Also, in this figure we have added the results of a calculation of $W(t)$ at times $t=2^{N}, N=0,1,2, \ldots$, according to the expression

$$
W\left(2^{N}\right)=\sum_{m=1}^{N} d_{N, m} .
$$

This confirms the value $s \cong 0.8001$.

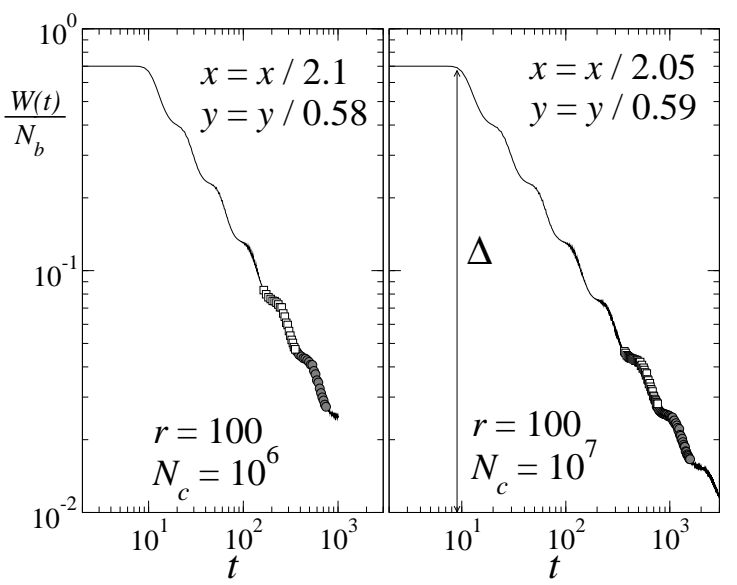

FIG. 11: The same as in the right panel of Fig. 10 but obtained with increased precision. In the left panel the number of initial conditions is $N_{c}=10^{6}$, while in the right panel $N_{c}=10^{7}$. The distance $\Delta$ is $\Delta=(1+|-1 / \alpha|) /(1+|-1|)$, where $\alpha$ is Feigenbaum's constant. This stems from the fact that all initial conditions out of the interval $(-1 / \alpha, 1)$ take a value inside this interval in the first iteration. As it can be observed the scaling factor for the horizontal axis converges to the exact value $x=2$.

\section{SUMMARY}

We have examined the process followed by an ensemble of uniformly distributed initial conditions $x_{0}$ across the phase space to arrive at the Feigenbaum attractor, or get captured by its corresponding repellor. Significantly, we have gained understanding concerning the dynamical ordering in the $x_{0}$, in relation to the construction of the families of phase-space gaps that support the attractor and repellor, and about the rate of approach of trajectories towards these multifractal sets, as measured by the fraction of bins $W(t)$ still occupied by trajectories at time $t$. An important factor in obtaining this knowledge has been the consideration of the equivalent dynamical properties for the supercycles of small periods in the bifurcation cascade [14].

As we have seen, a doubling of the period introduces welldefined additional elements in the hierarchy of the preimage structure, in the family of phase space gaps, and in the logperiodic power law decay of the rate $W(t)$. We have then 


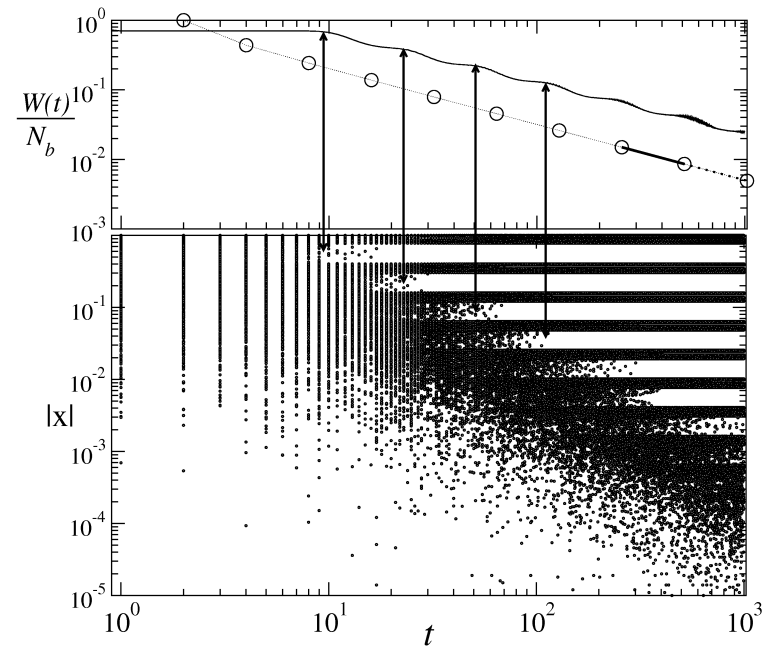

FIG. 12: Correspondence between the power-law decay with logperiodic oscillation features of the rate $W(t)$ with the sequential opening of phase space gaps. Top panel: The solid line is $W(t)$ from Fig. 9 and the open circles values are obtained for $W(t)$ from Eq. (3) at times $t=2^{N}, N=1,2, \ldots$ See text.

corroborated the wide-ranging correlation between time evolution at $\mu_{\infty}$ from $t=0$ up to $t \rightarrow \infty$ with the 'static' period- doubling cascade progression from $\mu=0$ up to $\mu_{\infty}$. As a result of this we have acquired an objective insight into the complex dynamical phenomena that fix the decay rate $W(t)$. We have clarified the genuine mechanism by means of which the discrete scale invariance implied by the log-periodic property in $W(t)$ arises, that is, we have seen how its self-similarity originates in the infinite hierarchy formed by the preimage structure of the attractor and repellor. The rate $W(t)$ can be obtained quantitatively (see Eq. (3)) from the supercycle diameters $d_{N, m}$. This basic data descriptive of the period-doubling route to chaos is also a sufficient ingredient in the determination of the anomalous sensitivity to initial conditions for the dynamics inside the Feigenbaum attractor [10].

The case is made in Ref. [12] that there is a statisticalmechanical property underlying the dynamics of an ensemble of trajectories en route to the Feigenbaum attractor (and repellor). There, Eq. (3) is identified as a partition function made up of $q$-exponential weighted configurations, while the fraction $W(t)$ of phase space still occupied at time $t$ is recognized as the $q$-exponential of a thermodynamic potential function. This is argued to be a clear manifestation of $q$-deformation of ordinary statistical mechanics where arguments can be made explicit [12].

Acknowledgments. Partial support by DGAPA-UNAM and CONACyT (Mexican Agencies) is acknowledged.
[1] See, for example, C. Beck, F. Schlogl, Thermodynamics of Chaotic Systems, Cambridge University Press, UK, 1993.

[2] H. Mori, H. Hata, T. Horita and T. Kobayashi, Prog. Theor. Phys. Suppl. 99, 1 (1989).

[3] See A. Robledo, Europhys. News 36, 214 (2005), and references therein.

[4] E. Mayoral, A. Robledo, Phys. Rev. E 72, 026209 (2005).

[5] G. Anania and A. Politi, Europhys. Lett. 7, 119 (1988).

[6] T.C. Halsey, M.H. Jensen, L.P. Kadanoff, I. Procaccia, B.I. Shraiman, Phys. Rev. A 33, 1141 (1986).

[7] J.-P. Eckmann and I. Procaccia, Phys. Rev. A 34, 659 (1986).

[8] M. Sano, S. Sato and Y. Yawada, Prog. Theor. Phys. 76, 945 (1986).

[9] Here we use the term $q$-deformed statistical mechanics with reference to the occurrence of statistical-mechanical expressions where instead of the ordinary exponential and logarithmic functions appear their $q$-deformed counterparts. The $q$ deformed exponential function is defined as $\exp _{q}(x) \equiv[1+$ $(1-q) x]^{1 /(1-q)}$, and its inverse, the $q$-deformed logarithmic function as $\ln _{q} y \equiv\left(y^{1-q}-1\right) /(1-q)$. The ordinary exponential and logarithmic functions are recovered when $q=1$.

[10] A. Robledo, Physica A 370, 449 (2006).

[11] C. Tsallis, J. Stat. Phys. 52, 479 (1988).

[12] A. Robledo, arXiv:0710.1047 [cond-mat.stat-mech].

[13] F. Baldovin and A. Robledo, Phys. Rev. E 72, 066213 (2005).

[14] A. Robledo and L.G. Moyano, Physical Review E 77, 032613 (2008); L.G. Moyano, D. Silva and A. Robledo, Cent. Eur. J. Phys. (in press); arXiv:0706.4415 [cond-mat,stat-mech].

[15] F.A.B.F. de Moura, U. Tirnakli and M.L. Lyra, Phys. Rev. E 62 6361 (2000).

[16] H.G. Schuster, Deterministic Chaos. An Introduction, 2nd Revised Edition (VCH Publishers, Weinheim, 1988).

[17] D. Sornette, Phys. Rep. 297, 239 (1998).

[18] P. Grassberger, Phys. Rev. Lett. 95, 140601 (2005).

[19] Evidently, the cantor set construction of the Feigenbaum attractor is comparable to our description of consecutive gap formation. 